\title{
Evaluation of lacrimal drainage system in Pseudoexfoliation syndrome
}

\author{
Fatma Corak Eroglu (D) ${ }^{1 凶}$, Mehmet Ali Sekeroglu (D) ${ }^{1}$, Tugce Horozoglu Ceran (D) ${ }^{1}$, Mert Simsek (D) ${ }^{1}$ and Gozde Hondur (D) \\ (c) The Author(s), under exclusive licence to The Royal College of Ophthalmologists 2021
}

PURPOSE: To evaluate the prevalence of the obstruction of lacrimal drainage system (LDS) in patients with pseudoexfoliation (PXF) syndrome.

MATERIALS AND METHODS: This cross-sectional study included 152 eyes of 76 consecutive patients with bilateral PXF syndrome and 170 eyes of 85 age and gender-matched controls. The LDS evaluation was performed based on dye disappearance test, slitlamp examination, diagnostic probing, and irrigation test. The presence of punctal stenosis and canalicular obstruction were considered as the obstruction of proximal LDS; and complete or incomplete nasolacrimal duct obstruction was considered as obstruction of distal LDS. Demographic characteristics, ophthalmologic findings, and prevalence and site of obstruction of LDS were compared among the groups.

RESULTS: The prevalence of obstruction of LDS was higher in the PXF syndrome group when compared to controls (21.1\% vs $12.2 \%)$, but the difference was not statistically significant $(p=0.061)$. The obstruction of proximal LDS was found to be more frequent in the PXF syndrome (17.7\%) group when compared to controls (10.0\%), and this difference was statistically significant $(p=0.041)$. There was significantly more punctal stenosis in the PXF syndrome group when compared to controls $(15.1 \%$ vs $7.6 \%$, $p=0.033)$. The prevalence of canalicular stenosis and obstruction of distal LDS was similar in the PXF and the control groups ( $p=0.596$ and $p=0.741$, respectively).

CONCLUSION: The prevalence of punctal stenosis was statistically significantly higher in the PXF syndrome group when compared to the controls. This association is probably related to increased local ocular surface inflammation which is triggered by the accumulation of PXF material.

Eye (2022) 36:2094-2098; https://doi.org/10.1038/s41433-021-01799-1

\section{INTRODUCTION}

Obstruction of lacrimal drainage system (LDS) is one of the most frequently encountered disorders in ophthalmology clinics, especially among the elderly population [1, 2]. Various pathogenic factors such as infection, trauma, systemic or topical drug toxicity, and inflammatory conditions originated from conjunctival and lacrimal system mucosa have been proposed to be associated with obstruction of LDS [1-3], due to swelling of the lacrimal system mucosa and subsequent fibrosis, finally leading to narrowing or obstruction of LDS [4-6]. The proximal LDS (punctum and canaliculus) is particularly vulnerable to various noxious exogenous or endogenous stimuli that could initiate the inflammatory process, which in turn plays a triggering role for the obstruction of LDS $[4,5]$.

Pseudoexfoliation (PXF) syndrome is a systemic disorder of the extracellular matrix that is characterized by the production and accumulation of abnormal fibrillar extracellular material within intraocular, periocular and even extraocular structures, including the blood vessels, skin, and visceral organs $[7,8]$. PXF syndrome is typically observed in individuals over the age of 50 years, and its frequency increases with aging [7]. Although the exact pathophysiological mechanism remained elusive, there is evidence that increased oxidative stress and low-grade inflammation caused by free radicals play a role in the pathogenesis of PXF syndrome [9-11]. PXF material in these patients is known to be present in all parts of the anterior segment as well as periorbital tissues such as conjunctiva, extraocular muscles, orbital septa, and Tenon's capsule [12]. Conjunctival accumulation of PXF material has also been associated with higher tear osmolarity, which can be responsible for perpetuating the inflammatory process in the tears and ocular surface [13].

PXF syndrome is well known to be associated with many ocular conditions such as glaucoma, keratopathy, zonulopathy, cataract formation, and dry eye syndrome [7-9]. However, to the best of our knowledge, the association between obstruction of LDS and PXF syndrome has not been studied before. In light of the fact that the prevalence of both diseases increases with aging, the presence of PXF material in the periocular tissues, and the wellknown role of the inflammation process in the pathogenesis of both diseases, the aim of the present study was to evaluate the prevalence of obstruction of LDS among patients with PXF syndrome and to compare these data with those of age-matched healthy control subjects.

${ }^{1}$ University of Health Sciences, Ulucanlar Eye Education and Research Hospital, Department of Ophthalmology, Ankara, Turkey. ${ }^{凶}$ email: dr_fatoscorak@hotmail.com 


\section{MATERIALS AND METHODS}

This cross-sectional study was conducted between February and April 2021 at a tertiary care ophthalmology clinic. Written informed consent was obtained from each participant and all procedures were in compliance with the tenets of the Declaration of Helsinki. Local ethics committee approval was received for the study.

Consecutive patients with bilateral PXF syndrome who were admitted for routine eye examination and consecutive healthy control subjects who were older than 50 years were recruited for the study. A total of 152 eyes of 76 patients with bilateral PXF syndrome and 170 eyes of 85 control subjects were included in this study.

All participants underwent a detailed ophthalmological examination including best-corrected visual acuity (BCVA) testing with Snellen chart, intraocular pressure (IOP) measurement with applanation tonometry, anterior segment examination with slit-lamp biomicroscopy, and dilated fundus examination. The diagnosis of PXF syndrome was based on the detection of PXF material at the pupillary margin and/or lens capsule on dilated slit-lamp biomicroscopic examination, as previously described in the literature $[7,8]$. Following a detailed ophthalmological examination, we also comprehensively reviewed the systemic and ocular history of the participants. The patients with any history of systemic diseases other than controlled hypertension were excluded from the study. The patients with a history or the evidence of any ocular surgery, trauma, disorders such as glaucoma, dry eye syndrome, blepharitis, uveitis, congenital lacrimal system disorders, and eyelid malposition were excluded from the study. We also excluded the patients who have formerly used topical antiglaucomatous agents and/or artificial tear preparations.

LDS evaluation was performed for all participants by an oculoplastic surgeon (FCE) who was blinded to the clinical data of the patients. The diagnosis of punctal stenosis was made based on the dye disappearance test (dye clearance time $>5 \mathrm{~min}$ ), slit-lamp examination (external punctal opening size less than $0.2 \mathrm{~mm}$ ), and the inability of the 27 -gauge lacrimal cannula to pass through the punctal without punctal dilatation [14, 15]. Following punctal dilation, prognostic probing was performed by $\# 00$ Bowman probe to evaluate the canalicular system patency. While a hard stop was defined as a patent canaliculus, a soft resistance to the probe that could not be overcome was described as canalicular system obstruction. The presence of punctal stenosis and/or canalicular obstruction were considered as obstruction of proximal LDS. Lacrimal irrigation with $2 \mathrm{ml}$ saline was performed to evaluate the distal LDS. The lacrimal cannula was passed through the lower punctum into the lacrimal sac and the nasolacrimal duct (NLD) was irrigated. Complete passage of fluid was defined as a patent distal LDS. A complete NLD obstruction was the diagnosis if fluid refluxed totally through the other punctum, while an incomplete NLD was the diagnosis if irrigation fluid passes into the nose as well as refluxing through the other punctum. The presence of complete or incomplete NLD obstruction was considered as obstruction of distal LDS.

\section{Statistical analysis}

Statistical analysis was performed using the statistical package SPSS version 22.0 (IBM Corporation, Armonk, NY, USA). Descriptive statistics, including the mean, standard deviation (SD), minimum-maximum, range, and percent (\%), were used for different variables. The Shapiro-Wilk test was performed to evaluate the normality of distribution. The difference between groups was evaluated using the independent $t$ test for the quantitative data, and the Pearson $\times 2$ and Fisher exact test for qualitative data. Pearson's correlation analysis was used to assess the correlation of the age and frequency of obstruction of LDS. A $p$ value smaller than 0.05 was considered as statistically significant.

\section{RESULTS}

One hundred and fifty-two eyes of 76 PXF syndrome patients (46 males, 30 females) with a mean age of $68.4 \pm 8.8$ years and 170 eyes of 85 control subjects ( 49 males, 36 females) with a mean age of $66.8 \pm 9.3$ years were recruited for the study. Both groups were age- and gender-matched (independent $t$ test, $p=0.249$, and Pearson $X 2$ test, $p=0.311$, respectively). There were no differences between the PXF syndrome and control groups in terms of BCVA and IOP ( $p>0.05$ for both). Demographic and clinical characteristics of patients are shown in Table 1.

The type of obstruction and the number of patients who have obstruction of LDS are shown in detail in Table 2. Although the prevalence of obstruction of LDS was higher in the PES group $(21.1 \%$ vs $12.2 \%)$, the difference was not statistically significant $(p=0.061)$. The obstruction of proximal LDS was more common among PXF syndrome group (17.7\%) as compared to controls $(10.0 \%)$, and this difference was statistically significant $(p=0.041)$. When the groups were compared in terms of site of obstruction of proximal LDS, there was significantly more punctal stenosis in the PXF syndrome group than controls $(p=0.033)$. The frequency of canalicular stenosis was similar in the PXF syndrome and the control groups $(p=0.569)$. The frequency of obstruction of distal LDS (complete/incomplete NLDO) was similar in the PXF syndrome and the control groups ( $p>0.05$ for both).

Although there was a moderate positive correlation between increasing age and frequency of punctal stenosis in the PXF syndrome group $(r=0.23, p=0.032)$, there was no correlation between age and canalicular stenosis and obstruction of distal LDS $(r=0.010, p=0.3$ and $r=0.011, p=0.4$, respectively). This correlation was statistically insignificant in the control group for punctal stenosis, canalicular stenosis and obstruction of distal LDS $(r=0.018, p=0.12 ; r=0.001, p=0.6$ and $r=0.002, p=0.5$, respectively).

\section{DISCUSSION}

PXF syndrome is well known to be associated with many ocular comorbidities including glaucoma, keratopathy, zonulopathy, cataract formation, and dry eye syndrome [7-9]. Both PXF syndrome and obstruction of LDS are closely related to inflammation, however, there is no data in the literature related to LDS in patients with PXF syndrome. In this study, we evaluated the LDS in patients with PES and demonstrated a preponderance of increased obstruction of LDS in PXF syndrome patients due to the affected proximal LDS. While the presence of the obstruction of distal LDS and canalicular obstruction were similar in PXF syndrome and control groups, the prevalence of punctal stenosis was statistically significantly higher in the patients with PXF syndrome compared to the controls.

Table 1. Clinical and demographic characteristics of patients.

\begin{tabular}{|llll} 
& PXF syndrome group (152 eyes of $\mathbf{7 6}$ patients) & Control group (170 eyes of 85 patients) & P \\
\hline Age (years), mean \pm SD (range) & $68.4 \pm 8.8(50-93)$ & $66.8 \pm 9.3(51-81)$ & \\
\hline Gender & & $49 / 85(57.6 \%)$ & $0.249^{*}$ \\
\hline Male & $46 / 76(60.5 \%)$ & $36 / 85(42.4 \%)$ & $0.311^{\dagger}$ \\
\hline Female & $30 / 76(39.5 \%)$ & $0.76 \pm 0.13$ & $0.681^{*}$ \\
\hline BCVA (Snellen Decimal) & $0.69 \pm 0.14$ & $16.8 \pm 2.9$ & $0.073^{*}$ \\
\hline IOP (mmHg) & $19.4 \pm 4.5$ &
\end{tabular}

${ }^{*}$ Independent sample-t test, ${ }^{+}$Pearson $\chi 2$ test. $P<0.05$ accepted as statistically significant.

PXF Pseudoexfoliation, BCVA Best corrected visual acuity (in Snellen decimals), IOP Intraocular pressure. 
Table 2. Comparison of PXF syndrome and control groups in terms of prevalence and type of obstruction of LDS.

\begin{tabular}{|c|c|c|c|}
\hline & PES group & Control group & $P$ \\
\hline Obstruction of LDS (total) & $32 / 152(21.1 \%)$ & $21 / 170(12.2 \%)$ & $0.061^{\dagger}$ \\
\hline Obstruction of proximal LDS & $27 / 152(17.7 \%)$ & $17 / 170(10.0 \%)$ & $0.041^{1}$ \\
\hline Punctal stenosis & $23 / 152(15.1 \%)$ & $13 / 170(7.6 \%)$ & $0.033^{\prime}$ \\
\hline Canalicular stenosis & $4 / 152(2.6 \%)$ & $4 / 170(2.4 \%)$ & $0.569^{\ddagger}$ \\
\hline Obstruction of distal LDS (NLDO) & $5 / 152(3.3 \%)$ & $4 / 170(2.4 \%)$ & $0.741^{\ddagger}$ \\
\hline Complete obstruction of distal LDS & $2 / 152(1.3 \%)$ & $2 / 170(1.2 \%)$ & $0.910^{\ddagger}$ \\
\hline Incomplete obstruction of distal LDS & $3 / 152(2 \%)$ & $2 / 170(1.2 \%)$ & $0.334^{\ddagger}$ \\
\hline
\end{tabular}

${ }^{\dagger}$ Pearson $\chi 2$ test, ${ }^{\ddagger}$ Fischer exact test. $P<0.05$ accepted as statistically significant and denoted in bold. PXF Pseudoexfoliation, LDS lacrimal drainage system, NLDO nasolacrimal duct obstruction.

PXF material is typically observed at the pupillary margin and anterior lens capsule. In addition to its typical intraocular localization, many studies in the literature reported the accumulation of PXF material in various extrabulbar tissues $[7,8]$. In an electron-microscopic study, Schlötzer-Schrehardt et al. [12]. demonstrated the accumulation of PXF fibres in limbal conjunctiva, extraocular muscles, orbital connective-tissue septa, and the walls of the posterior ciliary arteries, vortex veins, and central retinal vessels passing through the optic nerve sheaths and they stated that the close association of PXF aggregates with connective-tissue or muscle cells indicate their involvement in PXF fibre production. Therefore, it could be claimed that a variety of epithelial and mesenchymal cells may have common metabolic changes possibly associated with the aging process, resulting in the unregulated synthesis of microfibrillar components at multiple sites. Although there is no study investigating the presence of PXF material in LDS, in the light of the aforementioned data, it can theoretically be speculated that PXF material could accumulate in any site of the LDS (from punctum to NLD). However, this hypothesis may not be enough to explain our results showing that only punctal stenosis was more prevalent in the PXF syndrome group, whereas NLDO and canalicular obstruction were similar.

The punctum is the entry point for tears, and it is vulnerable to exogenous or endogenous irritants that could initiate the inflammatory process $[5,16]$. Inflammation, which is the physiological defence mechanism against various noxious stimuli, and subsequent fibrosis appear to be the currently proposed mechanism in the development of punctal stenosis [5, 16-19]. Various histopathological studies have reported that inflammation and/or fibrosis were the main histopathological features, with rates ranging from 73 to $100 \%$ in punctal stenosis specimens [15, 17-19]. There is plentiful evidence that cellular stress conditions caused by oxidative stress and low-grade inflammation constitute the main mechanism involved in the pathogenesis of the fibrotic process in PXF $[10,11,20-22]$. Our study demonstrated that there was an association between the obstruction of proximal LDS and PXF due to the predominance of punctal stenosis in patients with PXF syndrome. Consequently, increased low-grade chronic inflammation, which plays a role in the pathogenesis of both diseases, may be responsible for the relationship between PXF syndrome and punctal stenosis.

There are many studies in the literature investigating the tear film abnormalities, tear osmolarity, and conjunctival involvement in PXF [13, 23-25]. These studies suggested that accumulation of PXF material in the anterior segment causes conjunctival surface changes, decreased number of goblet cells, tear hyperosmolarity, and tear film instability. Hyperosmolar stress caused by loss of goblet cells and tear film instability promotes and maintains the local ocular inflammatory process [5, 13-26]. Tear osmolarity is considered the critical pathogenic factor that causes ocular surface inflammation, and hyperosmolarity is known to stimulate the production of inflammatory cytokines in tears and conjunctiva
[26-28]. Considering the punctum is the entry point for tears and is close to the conjunctiva, it could be assumed that prolonged ocular surface inflammation caused by PXF accumulation may play a pathogenic role in the development of punctal stenosis. Although many studies are investigating the presence of PXF material and related proteins in the aqueous humour [20,21, 29], only one recent study has studied the tear film concentration of clusterin, which co-localizes with fibrillar deposits in PXF material [30]. Their results implicated that the concentration of the clusterin, a protein associated with PXF material, was not different in PES than in control patients [31]. It can be assumed that the ocular surface inflammation in PXF is probably associated with oxidative stress triggering by the accumulation of PXF material in the anterior segment rather than the presence of PXF material itself in the tear film.

There was a moderate correlation between age and punctal stenosis in the PXF syndrome group in the current study. Many studies reported that the incidence of LDS obstruction increases with aging [1, 2]. Advanced age has been defined as the risk factor especially in punctal stenosis [1-5]. The association between age and punctal stenosis is considered to be the result of changes caused by the aging of the tissue surrounding the punctum and its fibrosis. PXF syndrome is known to be more common in the older population, and its frequency increases with aging [7, 8]. Local inflammation provoked by aging and distributed oxidative stress results in fibrous tissue production as a result. Therefore, another common feature shared by punctal stenosis and PXF syndrome is that the incidence of both diseases increases with advanced age.

The relationship of primary open-angle glaucoma and prolonged use of anti-glaucomatous medications with the obstruction of LDS has been previously reported in several studies [6, 31-33]. One of the strengths of the current study is the exclusion of patients with suspected/diagnosed glaucoma or who were using the anti-glaucomatous medications, as these may create confounding factors for obstruction of LDS in patients with PXF syndrome. Although this is the first study to evaluate the prevalence of obstruction of LDS among patients with PXF syndrome, the current study has some limitations. Conducting the study in a larger sample group would have provided more reliable results, but we had difficulty finding volunteers to participate in the study due to the COVID-19 pandemic. Another limitation is that the study group consisted of only bilateral PXF syndrome patients. A larger cohort of patients including affected and unaffected eyes in patients with unilateral PXF syndrome could be more enlightening about the relationship between obstruction of LDS and PXF. Although patients with dry eye syndrome and using artificial tear preparations were excluded from the study, the fact that the patients were not evaluated based on the Schirmer test, tear break-up time and corneal staining is another limitation of the study. Punctal stenosis could be related to a range of causes such as chronic blepharitis, eyelid malposition, 
and drugs [2-5]. It also shown that there was an association with canalicular stenosis [14]. Even though participants with chronic blepharitis, topical drug use, and eyelid malposition were excluded from our study, it may be difficult to distinguish whether punctal stenosis is an indirect association of ocular surface PXF or a direct manifestation of the syndrome. Therefore, our results showing that the prevalence of obstruction of proximal LDS was higher in the PXF syndrome group should be supported by an electron microscopic study in which PXF material will be investigated in samples obtained during lacrimal system surgery of PXF patients.

In conclusion, this study shows that patients with PXF syndrome are at risk of obstruction of proximal LDS due to punctal stenosis. This risk seems to be associated with local chronic inflammation triggered by PXF material. Physicians should be aware of this relationship for the early diagnosis and timely treatment of obstruction of LDS for prevention of the need for late lacrimal surgery.

\section{SUMMARY}

What was known before

- Pseudoexfoliation (PXF) syndrome is a systemic disorder of the extracellular matrix that is characterized by the production and accumulation of abnormal extracellular material within intraocular and periocular tissues.

- Although the PXF syndrome is well known to be associated with glaucoma, keratopathy, zonulopathy, and cataract formation, the association of obstruction of the lacrimal drainage system and PES association have not been studied before.

\section{What this study adds}

- Patients with PXF syndrome are at higher risk of obstruction of the proximal lacrimal drainage system.

- The higher prevalence of obstruction of the proximal lacrimal drainage system is probably related to local chronic inflammation triggered by the accumulation of PXF material.

\section{REFERENCES}

1. Das AV, Rath S, Naik MN, Ali MJ. The Incidence of lacrimal drainage disorders across a tertiary eye care network: Customization of an indigenously developed electronic medical record system-eyeSmart. Ophthalmic Plast Reconstr Surg. 2019;35:354-6.

2. Fiorino MG, Quaranta-Leoni C, Quaranta-Leoni FM Proximal lacrimal obstructions: a review. Acta Ophthalmol. (2021). https://doi.org/10.1111/aos.14762.

3. Schaefer DP Acquired etiologies of lacrimal system obstructions. In: Cohen A, Mercandetti M, Brazzo B (eds). The Lacrimal System. (Springer: Cham, 2015) 43-68.

4. Ali MJ Disorders of the upper lacrimal system. In: Ali M (eds). Principles and practice of lacrimal surgery. (Springer: Singapore, 2018) 135-40.

5. Starks VS, Yoon MK. Acquired obliteration of the proximal lacrimal drainage system. Ophthalmic Plast Reconstr Surg. 2019;35:342-5.

6. Pakdel F, Bahmani Kashkouli M. Lacrimal drainage obstruction associated with topical and systemic medications. J Ophthalmic Vis Res. 2009;4:270-1.

7. Schlötzer-Schrehardt U, Naumann GO. Ocular and systemic pseudoexfoliation syndrome. Am J Ophthalmol. 2006;141:921-37.

8. Ritch R. Ocular and systemic manifestations of exfoliation syndrome. J Glaucoma. 2014;23:S1-8.

9. Dursun F, Vural Ozec A, Aydin H, Topalkara A, Dursun A, Toker MI, et al. Total oxidative stress, paraoxonase and arylesterase levels at patients with pseudoexfoliation syndrome and pseudoexfoliative glaucoma. Int J Ophthalmol. 2015;8:985-90.

10. Tetikoglu M, Sagdik HM, Aktas S, Uçar F, Özcura F. Serum prolidase activity and oxidative stress in patients with pseudoexfoliation syndrome. Graefes Arch Clin Exp Ophthalmol. 2016;254:1339-43.
11. Yildirim Z, Yildirim F, Uçgun NI, Sepici-Dinçel A. The role of the cytokines in the pathogenesis of pseudoexfoliation syndrome. Int J Ophthalmol. 2013;6:505-3.

12. Schlötzer-Schrehardt U, Küchle M, Naumann GO. Electron-microscopic identification of pseudoexfoliation material in extrabulbar tissue. Arch Ophthalmol. 1991;109:565-70.

13. Öncel BA, Pinarci E, Akova YA. Tear osmolarity in unilateral pseudoexfoliation syndrome. Clin Exp Optom. 2012;95:506-9.

14. Kashkouli MB, Beigi B, Murthy R, Astbury N. Acquired external punctal stenosis: etiology and associated findings. Am J Ophthalmol. 2003;136:1079-84.

15. Hur MC, Jin SW, Roh MS, Jeong WJ, Ryu WY, Kwon YH, et al. Classification of lacrimal punctal stenosis and its related histopathological feature in patients with epiphora. Korean J Ophthalmol. 2017;31:375-82.

16. Ali MJ, Mishra DK, Baig F, Lakshman M, Naik MN. Punctal stenosis: histopathology, immunology, and electron microscopic features-a step toward unraveling the mysterious etiopathogenesis. Ophthalmic Plast Reconstr Surg. 2015;31:98-102.

17. Port AD, Chen YT, Lelli GJ Jr. Histopathologic changes in punctal stenosis. Ophthalmic Plast Reconstr Surg. 2013;29:201-4.

18. Jang JK, Lee SM, Lew H. A histopathological study of lacrimal puncta in patients with primary punctal stenosis. Graefes Arch Clin Exp Ophthalmol. 2020;258:201-7.

19. Reddy AK, Baker MS, Maltry AC, Maltry AC, Syed NA, Allen RC. Immunopathology and histopathology of conjunctival biopsies in patients with presumed idiopathic punctal stenosis. Br J Ophthalmol. 2017;101:213-7.

20. Botling Taube A, Konzer A, Alm A, Bergquist J. Proteomic analysis of the aqueous humour in eyes with pseudoexfoliation syndrome. $\mathrm{Br} J$ Ophthalmol. 2019;103:1190-4.

21. Ferreira SM, Lerner SF, Brunzini R, Evelson PA, Llesuy SF. Antioxidant status in the aqueous humour of patients with glaucoma associated with exfoliation syndrome. Eye. 2009;23:1691-7.

22. Schlötzer-Schrehardt U. Oxidative stress and pseudoexfoliation glaucoma. Klin Monbl Augenheilkd. 2010;227:108-13.

23. Kozobolis VP, Detorakis ET, Tsopakis GM, Pallikaris IG. Evaluation of tear secretion and tear film stability in pseudoexfoliation syndrome. Acta Ophthalmol Scand. 1999;77:406-9.

24. Kozobolis VP, Christodoulakis EV, Naoumidi II, Siganos CS, Detorakis ET, Pallikaris LG. Study of conjunctival goblet cell morphology and tear film stability in pseudoexfoliation syndrome. Graefes Arch Clin Exp Ophthalmol. 2004;242:478-83.

25. Erdoğan H, Arici DS, Toker MI, Arici MK, Fariz G, Topalkara A. Conjunctival impression cytology in pseudoexfoliative glaucoma and pseudoexfoliation syndrome. Clin Exp Ophthalmol. 2006;34:108-13.

26. Luo L, Li DQ, Pflugfelder SC. Hyperosmolarity-induced apoptosis in human corneal epithelialcells is mediated by cytochrome $c$ and MAPK pathways. Cornea. 2007;26:452-60.

27. Luo L, Li DQ, Doshi A, Farley W, Corrales RM, Pflugfelder SC. Experimental dry eye stimulates production of inflammatory cytokines and MMP-9 and activates MAPK signaling pathways on the ocular surface. Invest Ophthalmol Vis Sci. 2004;45:4293-301.

28. Fogagnolo P, Torregrossa G, Tranchina L, Ferreras A, De Cillá S, Labbé A, et al. Tear film osmolarity, ocular surface disease and glaucoma: a review. Curr Med Chem. 2019;26:4241-52

29. Berlau J, Lorenz P, Beck R, Makovitzky J, Schlötzer-Schrehardt U, Thiesen HJ, et al. Analysis of aqueous humour proteins of eyes with and without pseudoexfoliation syndrome. Graefes Arch Clin Exp Ophthalmol. 2001;239:743-6.

30. Can Demirdöğen B, Demirkaya-Budak S, Özge G, Mumcuoğlu T. Evaluation of tear fluid and aqueous humor concentration of clusterin as biomarkers for early diagnosis of pseudoexfoliation syndrome and pseudoexfoliative glaucoma. Curr Eye Res. 2020;45:805-13.

31. Kashkouli MB, Rezaee R, Nilforoushan N, Salimi S, Foroutan A, Naseripour M. Topical antiglaucoma medications and lacrimal drainage system obstruction. Ophthalmic Plast Reconstr Surg. 2008;24:172-5.

32. Kashkouli MB, Pakdel F, Hashemi M, Ghaempanah MJ, Rezaee R, Kaghaz-Kanani R, et al. Comparing anatomical pattern of topical anti-glaucoma medications associated lacrimal obstruction with a control group. Orbit. 2010;29:65-69.

33. Ortiz-Basso T, Galmarini A, Vigo RL, Gonzalez-Barlatay JM, Premoli EJ. The relationship between topical anti-glaucoma medications and the development of lacrimal drainage system obstruction. Arq Bras Ophtalmol. 2018;81:490-3.

\section{AUTHOR CONTRIBUTIONS}

All mentioned authors contributed to the study conception and design. Material preparation, data collection, and analysis were performed by FCE and MAS. The first draft of the manuscript was written by FCE, and all authors commented on previous versions of the manuscript. All authors read and approved the final manuscript. 
COMPETING INTERESTS

The authors declare no competing interests.

\section{ETHICS APPROVAL}

Local ethics committee approval was received for the study (Ankara Training and Research Hospital, Ankara, Turkey; project registration number: E-21-675). All protocols adhered to the Declaration of Helsinki.

\section{ADDITIONAL INFORMATION}

Correspondence and requests for materials should be addressed to Fatma Corak Eroglu.

Reprints and permission information is available at http://www.nature.com/ reprints

Publisher's note Springer Nature remains neutral with regard to jurisdictional claims in published maps and institutional affiliations. 